\title{
Algoritmo Evolutivo Eficiente Aplicado a la Planeación de la Expansión de Sistemas de Distribución
}

\author{
Carlos García $^{(1)}$, Edwin García ${ }^{(2)}$ y Fernando Villada(2) \\ (1) Empresas Públicas de Medellín, Carrera 58 No. 42-125, Oficina 8-332, Medellín-Colombia \\ (e-mail: carlos.garcía.montoya@epm.com.co) \\ (2) Universidad de Antioquia, Facultad de Ingeniería, Departamento de Ingeniería Eléctrica, \\ Calle 67 No. 53-108, Oficina 19-441, Medellín-Colombia (e-mail: egarciaq@udea.edu.co, \\ fvillada@udea.edu.co)
}

Recibido Nov. 02, 2011; Aceptado Ene. 12, 2012; Versión final recibida Ene. 30, 2012

\section{Resumen}

En este trabajo se aborda el problema de la optimización de la planeación de la expansión de un sistema de distribución real de la ciudad de Medellín (Colombia). El sistema de distribución en cuestión cuenta con 155 nodos y 154 tramos a un nivel de voltaje de 13200 volts. Para este fin se aplican algoritmos evolutivos eficientes, con el objeto de observar las mejoras y el desempeño que estos presentan frente a los algoritmos evolutivos convencionales. Además se plantea un modelo de planeación de la distribución mono-etapa y mono-objetivo, que tiene como función objetivo la optimización de los costos de construcción y las pérdidas técnicas del sistema. Finalmente se analiza el impacto que tienen los operadores eficientes diseñados especialmente para optimización, así como el análisis de los costos obtenidos.

Palabras clave: planeación de la distribución, algoritmos evolutivos, algoritmos genéticos, optimización.

\section{Efficient Evolutionary Algorithm Applied to Planning of Distribution System Expansion}

\begin{abstract}
The optimization of the expansion planning of the real distribution system in the city of Medellin in Colombia, is analyzed in this work. The distribution system has 155 nodes and 154 branches at a voltage level of 13200 volts. Efficient evolutionary algorithms are applied, with the purpose of observing their improvements and performance when compared with conventional evolutionary algorithms. Besides, a mono-stage and mono-objective distribution planning model, which objective function is the optimization of construction costs and technical losses of the system is also studied. Finally, the impact of specially designed efficient operators for optimization is discussed and the estimated costs are analyzed.
\end{abstract}

Keywords: distribution system planning, evolutionary algorithms, genetic algorithms, optimization 


\section{INTRODUCCIÓN}

La planeación de la expansión de sistemas eléctricos de distribución es una de las actividades primordiales que ejecutan las empresas dedicadas a la distribución de energía eléctrica. Puede afirmarse, que el principal objetivo de esta actividad es asegurarse de disponer de capacidad suficiente para atender la demanda de energía que los clientes requieren en un momento dado, obviamente cumpliendo con estándares técnicos de diseño, construcción y de calidad (Anaut et al., 2009). Son diferentes los elementos que debe considerar la planeación de la distribución, entre ellos las cantidades y tipos de red que lo componen, las proyecciones de crecimiento de la demanda, subestaciones y sus capacidades disponibles, además de variables externas como el crecimiento del país, problemas de orden socioeconómico, entre otros (Wills, 2004). Aparte de los elementos citados, la cantidad de equipos y dispositivos que constituyen el sistema y los diferentes escenarios futuros que pueden plantearse permiten que la tarea del planeador pueda ser abordada desde diferentes ópticas o enfocarse en objetivos específicos.

Por lo anterior el presente trabajo está enfocado en la optimización de la planeación de la expansión, pero debido a que optimizar modelos tan complejos como el planteado para este estudio no puede realizarse de manera manual, incluso con herramienta computacional convencional suele requerir grandes inversiones de tiempo y de memoria, entonces se han empleado diferentes técnicas de solución, partiendo de las técnicas clásicas exactas de optimización hasta llegar a las modernas heurísticas de búsqueda. Entre ellas se encuentran: programación cuadrática, branch-and-bound, redes neuronales, sistemas expertos y algoritmos genéticos (Mori y Yamada, 2007; Hao et al.,2011), perfilándose estos últimos como los más adecuados por sus características, ya que permiten implementar funciones objetivo complejas y tienen la capacidad de acoplarse a la búsqueda en espacios de solución complejos, propiedades que posee el problema tratado en éste estudio y por lo cual se han elegido los AG como técnica de solución.

Para alcanzar esta meta, en la sección Algoritmos Evolutivos se presenta una introducción a la computación inspirada en los procesos evolutivos presentes en la naturaleza, la sección Formulación detalla el modelo empleado para la solución del problema tratado en el trabajo, la sección Algoritmos Implementados contiene la descripción de los algoritmos desarrollados, la sección Resultados y Discusión muestra los resultados obtenidos en la implementación de operadores genéticos eficientes, además de la discusión respectiva, y finalmente en la última sección se encuentran las conclusiones. Los sistemas de distribución reales están construidos por una gran variedad de elementos que pueden ser ensamblados de diferentes maneras; es por esto que los circuitos de distribución poseen una gran cantidad de diferentes tipos de construcción y posibles topologías (Willis, 2004).

\section{CASO DE ESTUDIO}

El diagrama topológico del caso de estudio sobre el cual se enfocó este trabajo se muestra en la figura 1 y los tipos de construcción se describen en la tabla 1, la cual contiene las características eléctricas, la capacidad en amperios de los conductores, el costo en millones por kilómetro de unidad construida, calibre de la fase, calibre del neutro, el material del conductor de fase y el aislamiento. El sistema elegido tiene como características más representativas su radialidad, la cantidad de rutas posibles y la diversidad de magnitud de cargas. Además de la información presentada en la tabla 1, para ejecutar el algoritmo es necesario contar con los datos asociados a cada tramo del alimentador que es motivo de estudio. En este caso se dispuso de los siguientes datos por tramo: nodos inicial y final, longitud en metros, demanda proyectada en el nodo final expresada en kilovatios y kilovars (kilovoltios reactivos), un código que identifica si el tramo era proyectado o existente y otro código que identifique el tipo de construcción empleada. Cabe anotar que previo a la ejecución del algoritmo no fue necesario ningún proceso de preparación de los datos ni renumeración de nodos, dado que en la implementación de los algoritmos se desarrolló una rutina que genera una matriz que posee las conexiones existentes en la solución, razón por la cual no se hace necesaria una preparación de los datos, facilitando así el uso de la información que reposa en las bases de datos sin ser manipulada. 
Tabla 1: Datos y características de las unidades constructivas consideradas en el trabajo.

\begin{tabular}{|c|c|c|c|c|c|c|c|c|}
\hline $\begin{array}{c}\text { Tipo de } \\
\text { construcción }\end{array}$ & $\begin{array}{c}\mathrm{R} \\
\text { [ohmios/km] }\end{array}$ & $\begin{array}{c}\mathrm{X} \\
\text { [ohmios/km] }\end{array}$ & $\begin{array}{c}\text { Capacidad } \\
\text { [Amperios] }\end{array}$ & $\begin{array}{c}\text { Costo } \\
\text { [millones/km] }\end{array}$ & $\begin{array}{c}\text { Calibre } \\
\text { fase }\end{array}$ & $\begin{array}{c}\text { Calibre } \\
\text { neutro }\end{array}$ & Material & Aislamiento \\
\hline 1 & 0,2367 & 0,2633 & 400 & 164,620 & 266,8 & $1 / 0$ & ACSR & Cubierto \\
\hline 2 & 0,2365 & 0,417 & 400 & 64,142 & 266,8 & $1 / 0$ & ACSR & Desnudo \\
\hline 3 & 0,2367 & 0,2632 & 400 & 164,620 & 266,8 & $4 / 0$ & ACSR & Cubierto \\
\hline 4 & 0,2368 & 0,4064 & 400 & 64,142 & 266,8 & $4 / 0$ & ACSR & Desnudo \\
\hline 5 & 0,1423 & 0,1415 & 310 & 202,858 & 350 & $1 / 0$ & ACSR & Aislado \\
\hline 6 & 0,1423 & 0,1415 & 310 & 219,683 & 350 & $1 / 0$ & CU & Aislado \\
\hline 7 & 0,5829 & 0,2953 & 150 & 134,720 & $1 / 0$ & $1 / 0$ & ACSR & Cubierto \\
\hline 8 & 0,5826 & 0,449 & 150 & 56,783 & $1 / 0$ & $1 / 0$ & ACSR & Desnudo \\
\hline 9 & 0,6013 & 0,1746 & 150 & 111,260 & $1 / 0$ & $1 / 0$ & CU & Aislado \\
\hline 10 & 0,2911 & 0,4228 & 230 & 64,142 & $4 / 0$ & $1 / 0$ & ACSR & Desnudo \\
\hline
\end{tabular}

Para una adecuada interpretación de la figura 1, los tramos proyectados han sido representados por líneas en rojo, continuas para una posible solución y discontinuas para aquellas que no se emplean en la solución graficada.

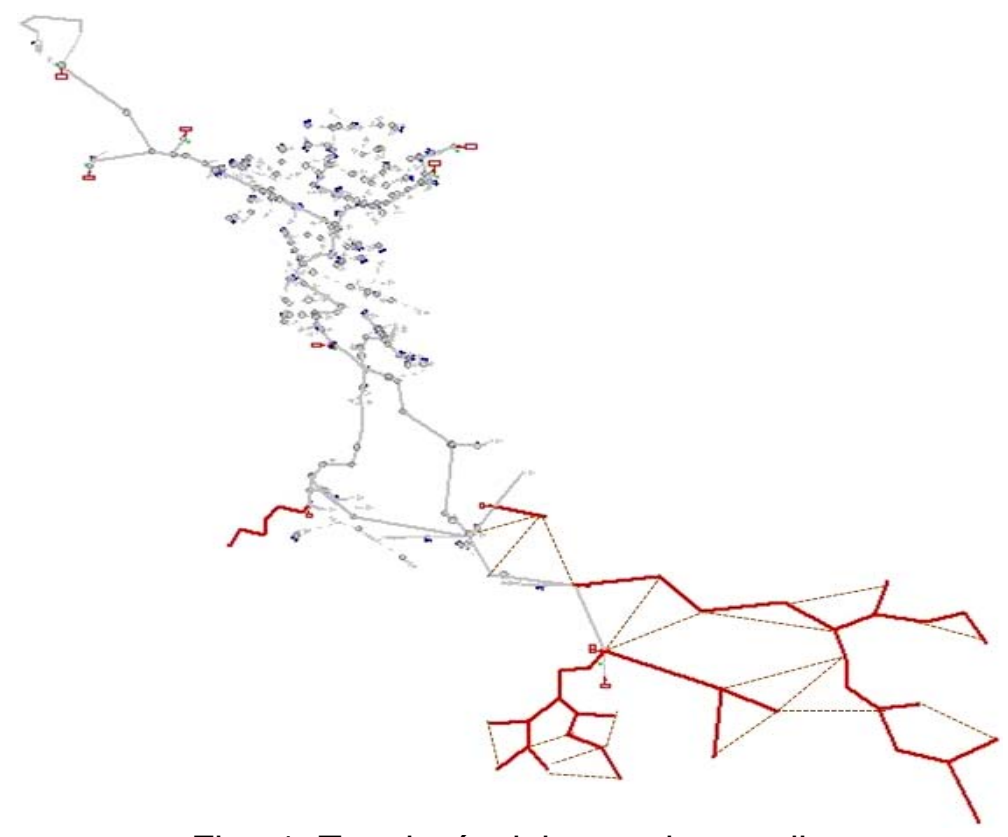

Fig. 1: Topología del caso de estudio

\section{MODELADO}

Antes de implementar algún algoritmo para resolver el problema que se está analizando, debe definirse un modelo que lo represente adecuadamente. Éste modelo está básicamente compuesto por: la o las funciones objetivo, la codificación y las restricciones que limitan el espacio de solución. El modelo propuesto aquí emplea una codificación no binaria y una función por agregación de costos lineal, la cual incluye los costos asociados a la construcción de nuevas redes y las pérdidas del sistema en un periodo de un año. La expresiones (1) a (4), presentan la función objetivo empleada y los elementos que la componen. Donde las ecuaciones (1)-(3) son propuestas en este trabajo enfocada a los resultados que se desean obtener respecto a la función de costos. La ecuación (4), por otra parte, es tomada de la referencia (Month, 2008).

$$
\begin{aligned}
& \mathrm{f}_{\text {costo }}=\sum_{\text {Tramo }=1}^{\mathrm{N}}\left(\mathrm{X}_{\mathrm{ij}} * \mathrm{C}_{\mathrm{fk}} * \mathrm{~L}_{\mathrm{ij}}\right) \\
& \mathrm{f}_{\text {cos-vble }}=\sum_{\text {Tramo }=1}^{\mathrm{N}}\left[\left(\mathrm{I}_{\mathrm{ij}}+\mathrm{I}_{\mathrm{ji}}\right)\left(\mathrm{X}_{\mathrm{ij}} * \mathrm{C}_{\mathrm{V}} * \mathrm{~L}_{\mathrm{ij}}\right)\right]
\end{aligned}
$$




$$
\begin{aligned}
& \mathrm{f}_{\text {Total }}=\mathrm{f}_{\text {costo }}+\mathrm{f}_{\text {cos-vble }} \\
& \mathrm{C}_{\mathrm{v}}=8760\left(\mathrm{C}_{\mathrm{kWh}}\right) \mathrm{F}_{\mathrm{p}} \mathrm{R}_{\mathrm{ij}} \frac{\mathrm{S}_{\mathrm{ij}}}{\mathrm{V}^{2}}
\end{aligned}
$$

Los componentes del modelo propuesto son: $f_{\text {costo }}$ es la función de costo de construcción; $f_{\text {cos-vble }}$ es la función de costo variable dependiente de las pérdidas; $f_{\text {Total }}$ es la función de costo total o función objetivo a optimizar; i es el nodo inicial; $j$ es el nodo final; $X_{i j}$ es el tramo entre los nodos i-j (Variable binaria); $L_{\mathrm{ij}}$ es la longitud del tramo entre los nodos $\mathrm{i}-\mathrm{j} ; \mathrm{C}_{\mathrm{fK}}$ son los costos fijos asociados a la construcción del tipo de red $\mathrm{K}$, el cual se muestra en la primera columna de la tabla $1 ; \mathrm{C}_{\vee}$ son los costos variables debidos a las pérdidas del sistema; $C_{k w h}$ son los costos por Kilovatio-hora; $F_{p}$ es el factor de pérdidas definido por el operador de red; $R_{i j}$ es la resistencia del conductor entre los nodos $\mathrm{i}-\mathrm{j} ; \mathrm{S}_{\mathrm{ij}}$ es la potencia que circula a través del conductor $\mathrm{i}-\mathrm{j}$; $\mathrm{V}$ es la tensión nominal del circuito; $\mathrm{l}_{\mathrm{ij}}$ es la corriente que circula por el tramo i-j.

Esta formulación se encuentra sujeta a un conjunto de restricciones que limitan la viabilidad de las soluciones evaluadas. Las restricciones consideradas en el modelo planteado son: a) Límites de capacidad de potencia en alimentadores y conductores, b) Estructura radial del sistema de distribución, c) Límites de tensión según la legislación actual, d) Ecuaciones de balance de potencia en los nodos, y e) Garantizar la entrega de potencia en todos los nodos proyectados dentro del sistema.

\section{ALGORTIMOS IMPLEMENTADOS}

El desarrollo del trabajo ha requerido de una etapa dedicada a la implementación de varios algoritmos, por supuesto con objetivos diferentes: uno genérico para resolver problemas de búsqueda u optimización de gran complejidad, otro para ejecutar el proceso de optimización y otro para la valoración de las soluciones posibles y la validación de restricciones definidas en la formulación. A continuación se describen estos algoritmos

\section{Algoritmos Evolutivos}

La computación evolutiva es parte de la Inteligencia Artificial, la cual es una rama de las ciencias de la computación (Haupt y Haupt, 2004). Ésta última, compuesta además por Redes Neuronales Artificiales y Sistemas Difusos, son técnicas que pretenden desarrollar agentes racionales no vivos, los cuales permiten analizar y/o resolver problemas de búsqueda u optimización de gran complejidad. Los algoritmos evolutivos (AE) siendo parte de la computación evolutiva, son diseñados para la solución de problemas, basándose en principios de la teoría evolutiva. Dentro de estos se ha desarrollado una amplia gama, todos bajo los mismos conceptos poblacionales como son: selección, recombinación y mutación. La selección es la parte del algoritmo que trata de reproducir la selección natural de los individuos de una población. La recombinación o cruce, se encarga de la reproducción de los individuos, y por último la mutación que pretende modelar los efectos producidos por este comportamiento en los genes de los individuos. Los AE más empleados son: los Algoritmos Genéticos (AG), Programación Evolutiva y Programación Genética (Mori y Yamada, 2007). Debido a su alta capacidad de búsqueda y fácil adaptabilidad, estos se han perfilado como aptos para su aplicación en diferentes problemas bajo investigación en la última década. Los AG ya han sido empleados en sistemas de potencia y de distribución (Anaut et al., 2009), logrando muy buenos resultados hasta convertirlos en excelentes candidatos para ser implementados en problemas como el planteado en el presente trabajo (Hao et al., 2011; Rajkumar et al., 2008).

\section{Algoritmo Genético}

El primer algoritmo implementado fue un AE canónico (Haupt y Haupt, 2004), con una población inicial constituida por individuos factibles evaluados previamente. Este algoritmo, se caracteriza 
por emplear estrategias de cruce y mutación convencionales como el cruce en dos puntos, y la mutación aleatoria, además de selección por torneo. Este algoritmo fue probado con el sistema de distribución presentado en la sección caso de estudio, evidenciando que, a pesar de los relativos buenos rendimientos obtenidos dentro de las simulaciones realizadas, el algoritmo presenta problemas de transferencia de buenas estructuras de una generación a otra, alta tasa de individuos infactibles y falta de diversidad en el conjunto final de soluciones (Cebrian y Kagan, 2010; Rajkumar et al., 2008).

Basado en los resultados y observaciones se encontró que estos problemas podrían disminuirse si se modifican los operadores genéticos del algoritmo canónico, por unos particulares que se adecuen al planteamiento del problema de la optimización de la planeación y garanticen que dentro del proceso se transmita la calidad de los individuos que se cruzan a las siguientes generaciones (Ferreira et al., 2001). Esta apreciación se materializó implementando un AG Modificado, el cual se caracteriza por poseer la misma estructura del canónico, pero empleando estrategias de mutación y cruce, que garantizan que los individuos que surgen reciben buenas características de los padres. Los resultados logrados mediante la implementación de las estrategias mencionadas pueden apreciarse en la sección resultados y discusión. La estructura de ambos algoritmos: el canónico y el modificado, está representada en la Fig. 2.

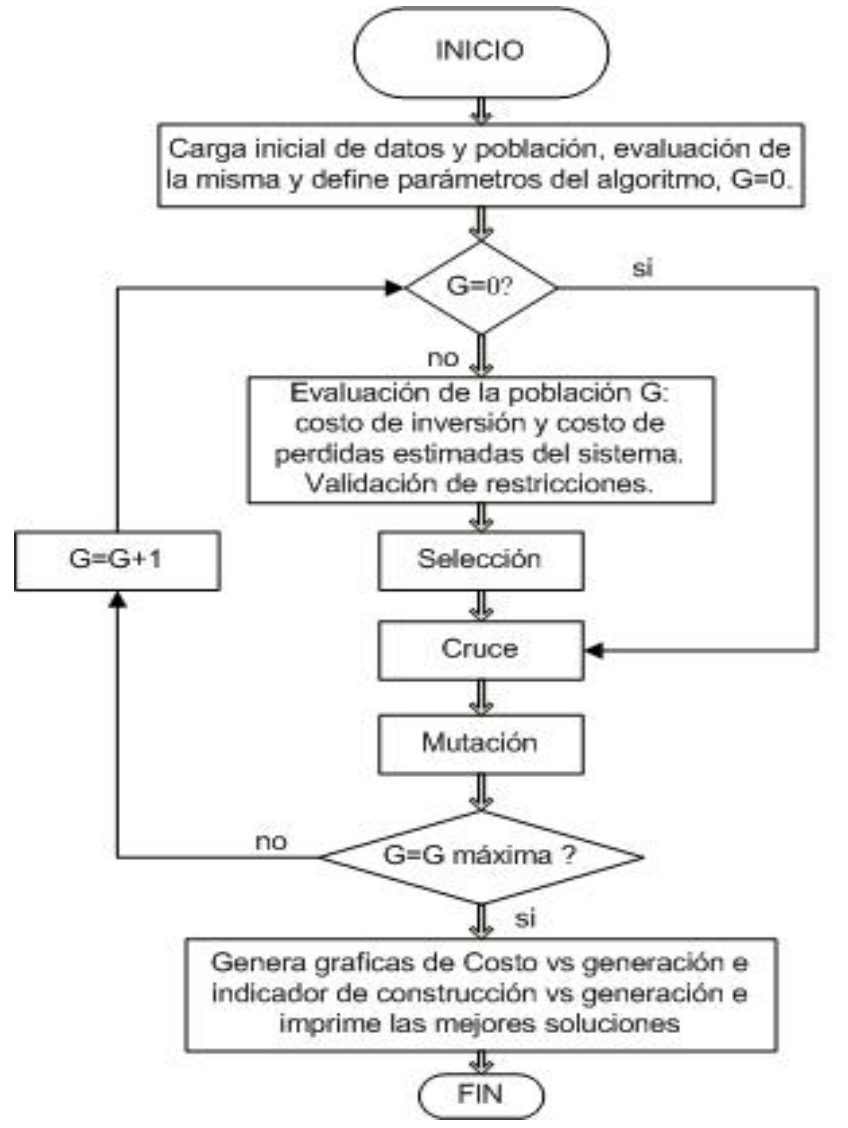

Fig. 2: Algoritmo Genético Mono Objetivo Implementado

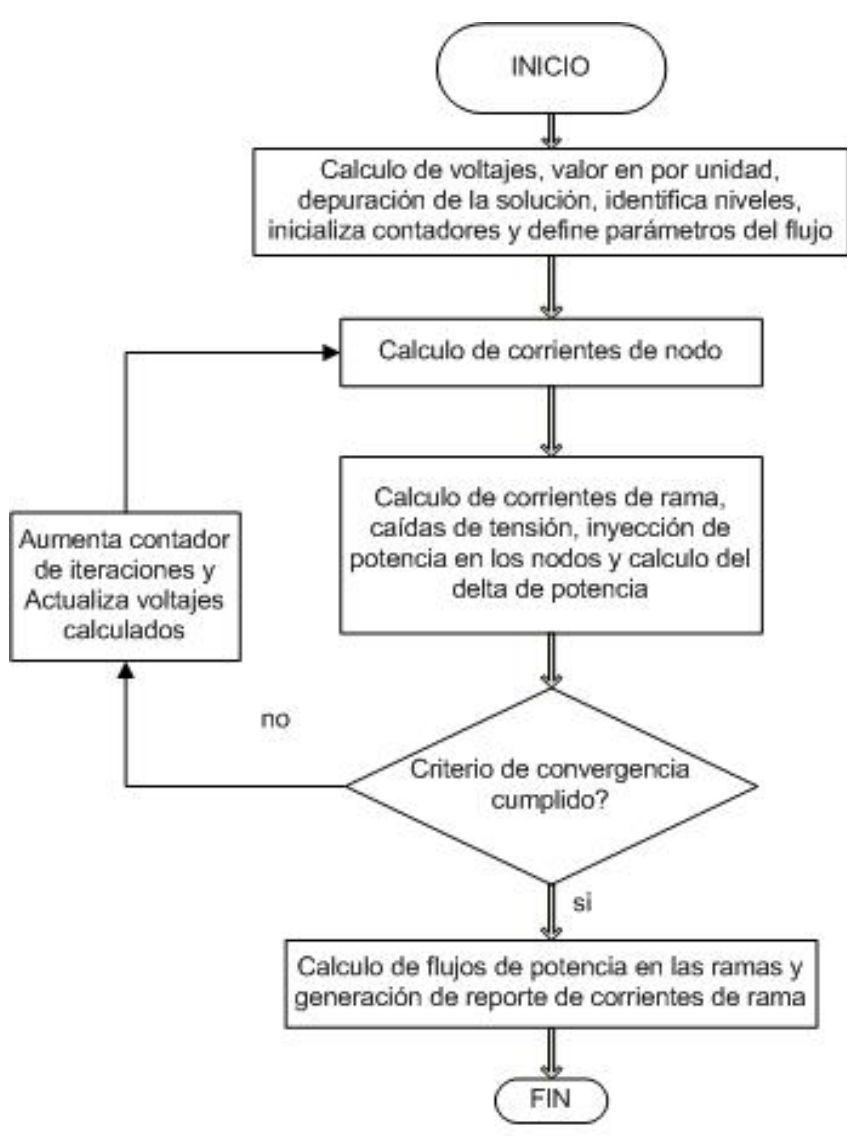

Fig. 3: Flujo Radial Implementado

\section{Algoritmo para Validación de Restricciones}

Dentro del proceso generacional, debe garantizarse que los individuos cumplen con las restricciones del problema, lo cual implica una validación de cada individuo. Esta evaluación es ejecutada mediante un algoritmo que verifica todas las restricciones definidas en la formulación. En el caso de las redes de distribución, la evaluación de las características técnicas del sistema se hace mediante la implementación de un flujo de carga, que en este caso particular es radial y balanceado, el cual puede apreciarse en la Fig. 3. 


\section{RESULTADOS Y DISCUSIÓN}

Para obtener los resultados se partió de la implementación de un algoritmo canónico, con el cual se ejecutaron diferentes simulaciones empleando el sistema descrito en la sección caso de estudio. Se realizaron diferentes pruebas, las cuales consistieron básicamente en variar el valor de la tasa de mutación y evaluar el desempeño de los algoritmos; validando convergencia, tiempos de ejecución y diversidad de las soluciones. Los valores de los parámetros empleados para las pruebas fueron: limite generacional 40 generaciones, población inicial 5 individuos, tasa de cruce $100 \%$ lo que representa que se ejecuta un cruce por cada individuo de la población general, y límite de sobrevivientes 10 individuos. Un resumen de resultados obtenidos en las pruebas efectuadas se observa en la tabla 2, dentro de la cual se especifica el valor en porcentaje de disminución del tiempo de ejecución logrado al cambiar las estrategias de los operadores del algoritmo canónico.

Tabla 2: Resultados pruebas de variación de la mutación

\begin{tabular}{|c|c|c|c|}
\hline \multirow{2}{*}{ Mutación [\%] } & \multicolumn{2}{|c|}{ Tiempo de ejecución [s] } & $\begin{array}{c}\text { Porcentaje de disminución de } \\
\text { tiempo }\end{array}$ \\
\cline { 2 - 3 } & AG Canónico & AG Modificado & $34 \%$ \\
\hline 10 & 15.2 & 10.1 & $33 \%$ \\
\hline 5 & 14.4 & 9.7 & $48 \%$ \\
\hline 4 & 18.9 & 9.8 & $46 \%$ \\
\hline 3 & 17.9 & 9.7 & \\
\hline
\end{tabular}

Pese a que los resultados obtenidos con el algoritmo canónico pueden considerarse relativamente buenos al estar frente a un sistema de distribución de pequeñas dimensiones, se evidencia el efecto que tiene la mutación sobre el desempeño del mismo, al afectar tanto la convergencia del algoritmo como la diversidad de las soluciones, características que son importantes al medir el desempeño, dado que de ellas depende la forma como el algoritmo explora el espacio de solución o si éste se queda atrapado en un óptimo local; estos comportamientos, la baja transferencia de buenas características hacia los descendientes de parte de los padres seleccionados (heredabilidad) y la poca diversidad que presentan los individuos cuando se emplean tasas de mutación bajas, fueron mejoradas notablemente al implementar los operadores genéticos del algoritmo modificado, alcanzando disminuciones en tiempo de ejecución de hasta $48 \%$ cuando se tiene una mutación del $4 \%$.

Las Fig. 4 y 5 muestran el desempeño de los algoritmos implementados empleando una tasa de mutación del $10 \%$. En éstas se observa claramente como el AG canónico no logra convergencia y se evidencia que para la misma tasa de mutación el efecto de convergencia es mejor sobre el AG modificado. También se aprecia en los resultados, como al disminuir la magnitud de la mutación, se aprecia como el AG modificado tiende a una convergencia más rápida, situación que obedece a que la diversidad de los individuos que integran la población es proporcional al valor de éste operador. EI AG canónico se ve afectado en mayor medida por este parámetro, ocasionando incluso que no logre convergencia, tal como se observa en los resultados de la Fig. 4. Este comportamiento puede ser confirmado analizando el desempeño del algoritmo observado en las Fig. 6 a 9. Las mejoras en el desempeño del AG Modificado se lograron gracias a que éste fue implementado con un cruce que garantiza la heredabilidad, transmitiendo las mejores estructuras entre padres y descendientes, garantizando de esta forma que tramos de red del sistema de distribución que ocasionan inviabilidad en la solución planteada fueran solucionadas antes de proceder con la evaluación. Estas porciones (o tramos de red) de la solución, son elegidas aleatoriamente de entre los dos individuos designados como padres, garantizando así su viabilidad y evitando emplear esfuerzos computacionales no necesarios, sobre soluciones que no son viables, y además se logra que el algoritmo explore controladamente dentro del espacio de solución infactible.

Además de la estrategia de cruce descrita, se implementó una mutación que identifica rutas alternativas para atender las cargas del sistema de distribución que no han sido empleadas en la 
solución a la cual se le está efectuando la mutación. La ruta alternativa identificada, reemplaza la existente en la solución, efectuando la mutación y garantizando la factibilidad del individuo. Evidentemente, gracias a las modificaciones realizadas, se logró superar ostensiblemente el desempeño del algoritmo canónico: alcanzando convergencia más rápida, diversidad en las soluciones y mejorando los problemas identificados dentro del AG canónico, todo esto sin cambiar su estructura, solo modificando las estrategias. Además, las figuras 4 a 9 muestran que al disminuir el porcentaje de mutación también se logra disminuir y estabilizar el costo de la solución más óptima, como se muestra en la tabla 3; alcanzándose asimismo una solución óptima ligeramente más económica para el AG modificado comparado con el AG canónico.

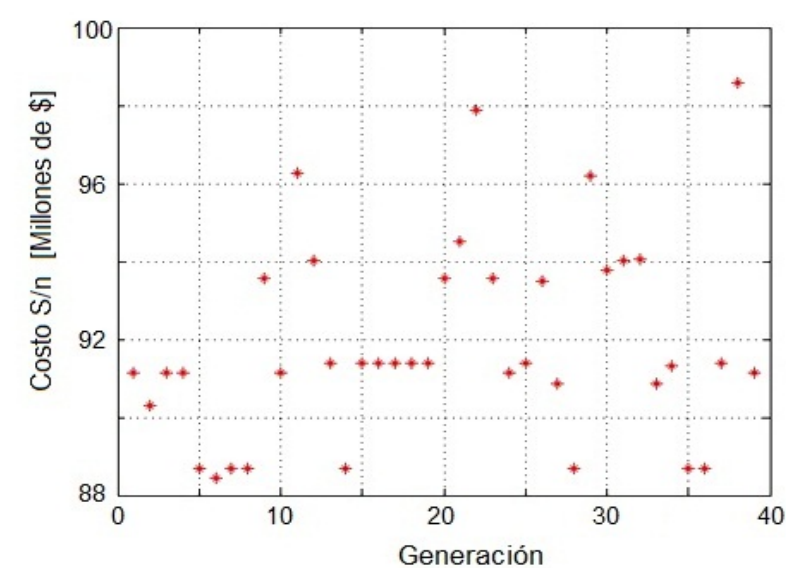

Fig. 4: Resultados con mutación $10 \%$ (AG Canónico)

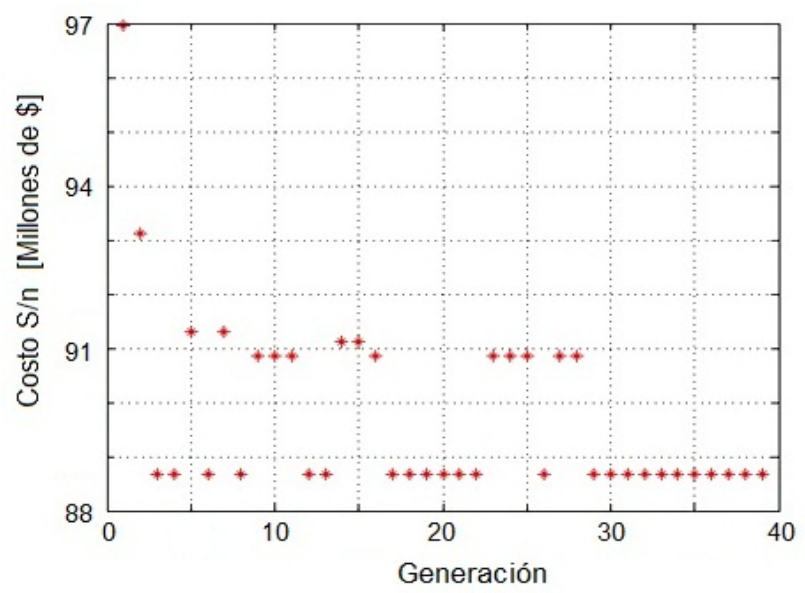

Fig. 6: Resultados con mutación $5 \%$ (AG Canónico)

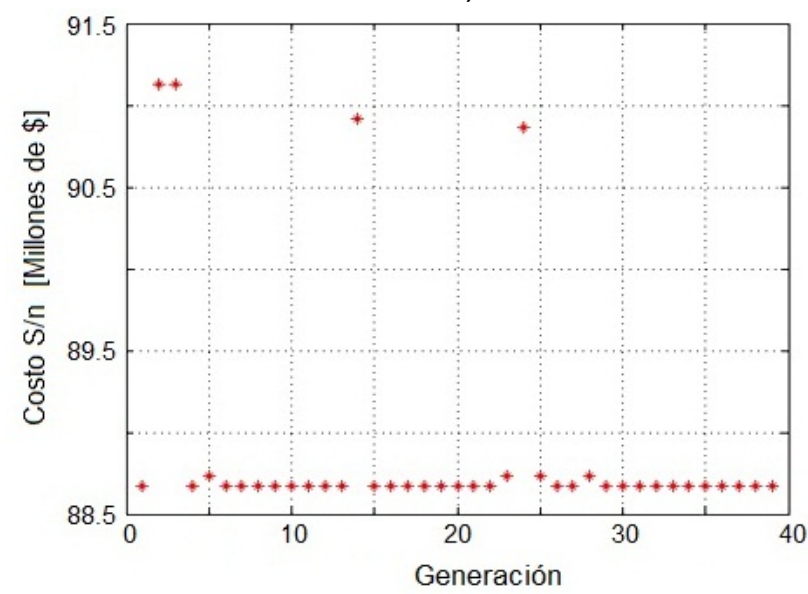

Fig. 8: Resultados con mutación 3 \% (AG Canónico)

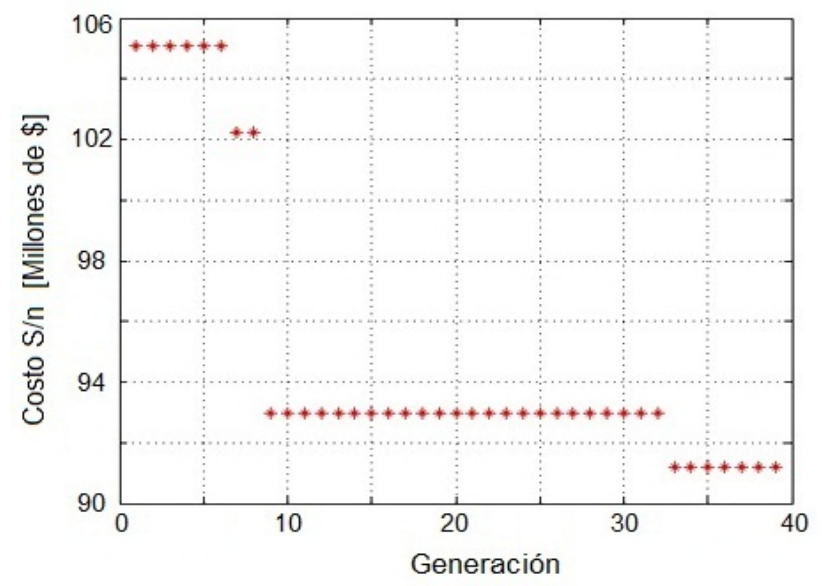

Fig. 5: Resultados con mutación 10 \% (AG Modificado)

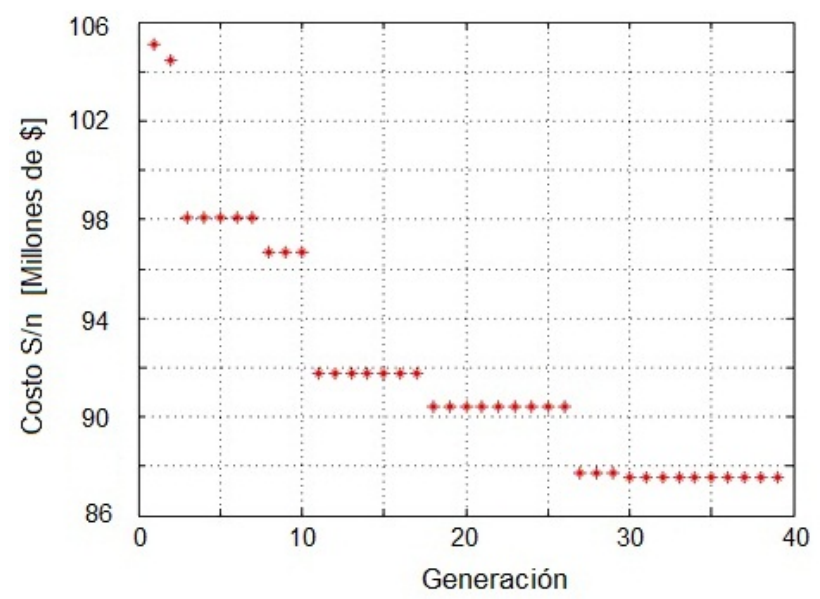

Fig. 7: Resultados con mutación 5 \% (AG Modificado)

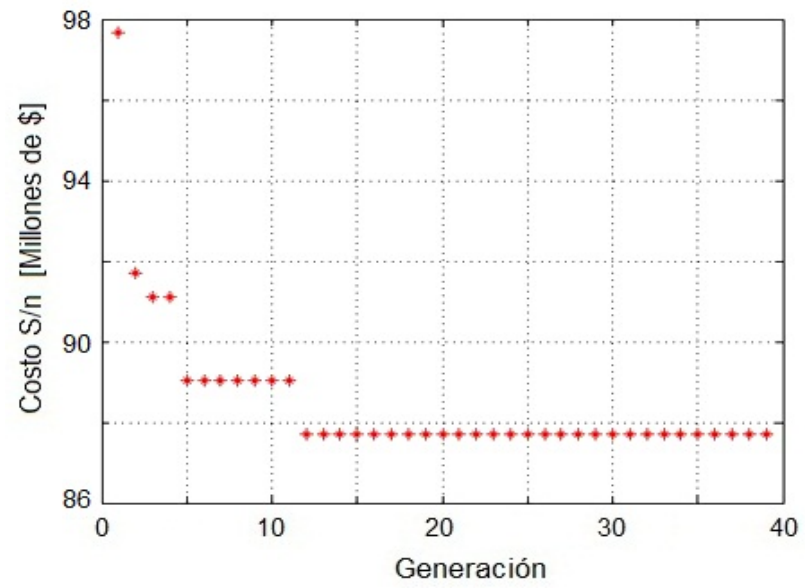

Fig. 9: Resultados con mutación 3 \% (AG Modificado) 
Tabla 3: Costo de la solución óptima respecto al porcentaje de mutación y al tipo de algoritmo genético implementado.

\begin{tabular}{|c|c|c|}
\hline \multirow{2}{*}{ Mutación [\%] } & \multicolumn{2}{|c|}{ Solución más económica (millones de pesos) } \\
\cline { 2 - 3 } & AG canónico & AG modificado \\
\hline $10 \%$ & 88.3 & 91.2 \\
\hline $5 \%$ & 88.7 & 87.5 \\
\hline $3 \%$ & 88.7 & 87.5 \\
\hline
\end{tabular}

\section{CONCLUSIONES}

Existe una relación directa entre los operadores genéticos, el tiempo de ejecución del algoritmo y los costos de las soluciones óptimas obtenidas, presentando un mejor comportamiento en estos aspectos el AG modificado (ver las tablas 2 y 3). Los AG canónicos son menos eficientes que los AG modificados en cuanto a esfuerzo computacional, dado que los operadores genéticos del primero no están diseñados para transmitir las buenas características genéticas de los padres hacia sus descendientes y, su desempeño y convergencia son altamente dependientes de la tasa de mutación. Los operadores genéticos que fueron implementados en el AG modificado mejoran notablemente su desempeño disminuyendo el peso computacional, lo cual es altamente conveniente considerando las dimensiones de los sistemas de distribución de energía. El AG modificado encuentra soluciones óptimas ligeramente más económicas que el AG canónico, lo cual también depende del porcentaje de mutación que se aplique.

\section{REFERENCIAS}

Anaut D. O., Di Mauro G. F., Meschino G. y Suárez J. A.; Optimización de Redes Eléctricas Mediante la Aplicación de Algoritmos Genéticos, Revista Información Tecnológica: 20(4), 137-148 (2009)

Cebrian J.C. y Kagan N.; Reconfiguration of distribution networks to minimize loss and disruption costs using genetic algorithms, Electric Power Systems Research: 80(1), 53-62 (2010)

Ferreira L.A.F.M., Carvalho P.M.S., Jorge L.A., Grave S.N.C. y Barruncho L.M.F.; Optimal distribution planning by evolutionary computation-how to make it work, Transmission and Distribution Conference and Exposition, IEEE/PES, 1, 469-475 (2001).

Hao Y., Li G., Kangning W. y Jinhao W; Multi-objective optimal power filter planning in distribution network based on fast nondominated sorting genetic algorithms, Electric Utility Deregulation and Restructuring and Power Technologies (DRPT), 4th International Conference, 234-240, 6-9 July (2011).

Haupt R. L. y Haupt S. E.; Practical Genetic Algorithms, John Wiley \& Song, $2^{\text {nd }}$ ed, Canada (2004). Month J. A., Electric power system applications of optimization, Editorial CRC Press, Estados Unidos (2008).

Mori H. y Yamada Y.; An Efficient Multi-objective Meta-heuristic Method for Distribution Network Expansion Planning, Power Tech, IEEE Lausanne: 374-379, 1-5 July (2007).

Rajkumar N., Vekara, T. y Alander J.; A Review of Genetic Algorithms in Power Engineering, 13th Finnish Artificial Intelligence Conference, 15-32, 20-22 August (2008).

Willis L.; Power Distribution Planning Reference Book, second edition, Marcel Dekker, Inc., North Carolina, United States (2004). 